\title{
Non-Conservative Evolution of Binary Stars
}

\author{
Christopher A. Tout \\ Institute of Astronomy, The Observatories, Madingley Road, Cambridge CB3 0HA, England \\ email: cat@ast.cam.ac.uk
}

\begin{abstract}
Various processes can lead to non-conservative evolution in binary stars. Under conservative mass transfer, both the total mass and the orbital angular momentum of the system are conserved. Thus, the transfer of angular momentum between the orbit and the spins of the stars can represent one such effect. Stars generally lose mass and angular momentum in a stellar wind so, even with no interaction, evolution is non-conservative. Indeed, a strong wind can actually drive mass transfer. During Roche lobe overflow itself, mass transfer becomes nonconservative when the companion cannot accrete all the material transferred by the donor. In some cases, material is simply temporarily stored in an accretion disc. In others, the companion may swell up and initiate common envelope evolution. Often the transferred material carries enough angular momentum to spin the companion up to break-up, at which point it could not accrete more. We investigate how this is alleviated by non-conservative evolution.
\end{abstract}

Keywords. binaries: close, stars: mass loss, planetary systems

In 1848, Édouard Roche formulated what came to be known as the Roche limit and began the theory of the Roche potentials. In the twentieth century, these became used to study the shapes of interacting binary stars and, with the explanation of the Algol paradox, that in a coeval binary the fainter component could be larger, redder and less massive than its companion (Hoyle 1955; and Crawford 1955), the concept of mass transfer by Roche lobe overflow became established. To understand how a mass-transferring close binary star evolves, we must know whether mass, and indeed angular momentum, are lost from the system in significant quantities during the mass transfer.

\section{Conservative Evolution}

Let us first define conservative mass transfer to be without mass loss or angular momentum loss from the system. That is, both the total mass and the total angular momentum are conserved. We shall restrict the definition further by assuming the stars behave as point masses, material flows between them uniformly over an orbital period, and no angular momentum can be stored in either the stars or circumstellar matter. For illustration, we shall consider circular orbits. Fig. 1 shows this simple set up in which the two stars with masses $M_{1}$ and $M_{2}$ orbit their centre of mass in circular orbits of radii $a_{1}$ and $a_{2}$ at angular velocity $\Omega$. The total mass of the system $M=M_{1}+M_{2}$ and the separation of the stars $a=a_{1}+a_{2}$, so that $a_{1}=a M_{2} / M$. The total orbital angular momentum is then

$$
J=\frac{M_{1} M_{2}}{M} a^{2} \Omega
$$

and the orbit obeys the generalised form of Kepler's third law

$$
\left(\frac{2 \pi}{P}\right)^{2}=\Omega^{2}=\frac{G M}{a^{3}},
$$

where $P$ is the orbital period and $G$ is Newton's gravitational constant. 


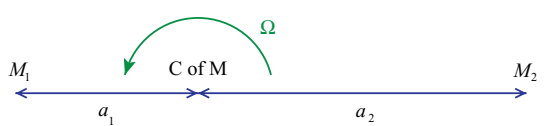

Figure 1. A simple binary star consisting of two point mass stars in a circular orbit.

For conservative evolution, we then take the rate of change of total mass $\dot{M}=0$ but allow mass transfer so that $\dot{M}_{2}=-\dot{M}_{1}$ and $\dot{J}=0$. Taking a logarithm of equation (1.1) and differentiating, we arrive at

$$
\frac{\dot{J}}{J}=\frac{\dot{M}_{1}}{M_{1}}+\frac{\dot{M}_{2}}{M_{2}}+2 \frac{\dot{a}}{a}+\frac{\dot{\Omega}}{\Omega}=0 .
$$

We do the same with Kepler's third law and integrate to obtain the familiar results

$$
P\left(M_{1} M_{2}\right)^{3}=\text { const and } \mathrm{a}\left(\mathrm{M}_{1} \mathrm{M}_{2}\right)^{2}=\text { const. }
$$

Thus, for mass transfer from star 1 to star 2 both the period and separation decrease while $M_{1}>M_{2}$, and subsequently increase.

\section{Non-Conservative Evolution}

When evolution is non-conservative, both $\dot{M}<0$ and $\dot{J}<0$. It is hard to envisage substantial mass loss without that mass carrying off angular momentum; and angular momentum cannot be lost without mass to transport it, though this mass may be very small in the case of magnetic braking or gravitational radiation. First, let us consider an isotropic stellar wind from one of our two point masses, star 1, when there is no mass transfer. So, star 1 loses mass at a rate $-\dot{M}=-\dot{M}_{1}$. This mass carries away its specific orbital angular momentum so that

$$
\dot{J}=\dot{M} a_{1}^{2} \Omega
$$

We can divide this by $J$ and proceed as before to obtain the, again familiar, results

$$
P M^{2}=\text { const } \text { and } a M=\text { const. }
$$

So, the period and separation increase. The solar system will grow as the Sun loses mass.

\section{Stable Roche Lobe Overflow}

Fig. 2 shows a star that only slightly overfills its Roche lobe. Subsonic flow accelerates to supersonic as it passes through the rocket-like nozzle at the $L_{1}$ point. The ballistic stream this creates is then confined to the Roche lobe of its companion. If the stream hits the companion before encircling it, material may accrete directly. If the companion is too small so that the stream has too much angular momentum to fall on directly, it encircles the star, collides with itself and spreads out to form an accretion disc. Viscosity in the disc allows a small amount of the mass to carry angular momentum outwards while most of the mass drifts inwards. The disc is truncated by tides within the Roche lobe of the accreting star and these same tides return angular momentum to the orbit. Thus, we do not expect material flowing through the $L_{1}$ point to be lost during the overflow. Any material that is lost must be driven away by some energy source associated with the secondary star or the accretion flow on to it.

However there is, at least circumstantial, evidence that binary systems do not evolve conservatively. All Algol-like systems observed today have mass ratios that are significantly inverted, in that the mass of the star that evolved first, and so was originally 


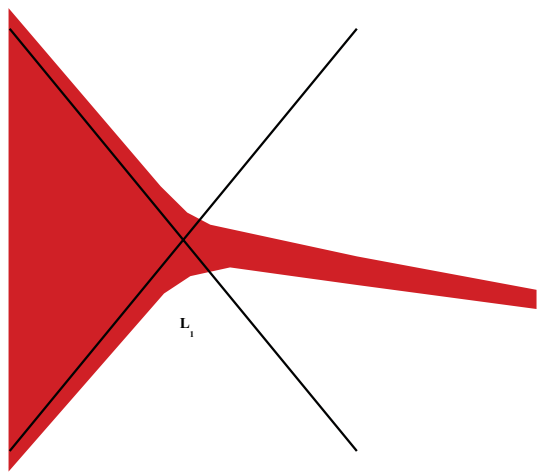

Figure 2. The region of the inner Lagrangian point $L_{1}$ for a star that only just overfills its Roche lobe. The equipotential surfaces at the $L_{1}$ point form a rocket-like nozzle through which material flows. On the left flow remains subsonic, so that the star can be treated as if in hydrostatic equilibrium, while on the right flow is supersonic and so ballistic.

the more massive, is now somewhat less than that of its accreting companion. Morton (1960) deduced that this meant that all Algols should have gone through a rapid mass transfer phase that was very short-lived compared to the state in which we observe them now. This can be explained if the initially more massive star first filled its Roche lobe in the Hertzsprung gap, as a subgiant, when loss of mass would lead the star to expand and transfer mass on a thermal timescale. Even then, the process of overflow ought to be conservative except that the secondary star cannot itself accrete the material fast enough. Indeed, Paczyński \& Ziółkowski (1967) found that the resulting Algol systems are more realistic if half the mass transferred is actually lost from the system.

The need for angular momentum loss from binary stars is confirmed by the cataclysmic variables. Most of these consist of a low-mass red dwarf transferring mass to a somewhat more massive white dwarf. The red dwarf evolves far too slowly to be driving the mass transfer by its own nuclear evolution, and simple mass loss alone would widen rather than shrink the orbit. Indeed, the white dwarf in these systems must have evolved as the core of a red giant whose entire envelope has been lost from the system.

\section{The Spins of the Stars}

Before considering true non-conservative evolution, let us first consider relaxing the assumption that the stars are point masses. As a star grows to fill its Roche lobe, tides tend to lock its spin with the orbit, and angular momentum flows between the star and the orbit. Typically, a giant star has spun down as it expanded and so requires more angular momentum to spin it up. From Kepler's third law and our expression for orbital angular momentum (equation 1.1), transfer of angular momentum from the orbit to the star shrinks the orbit and so increases its angular velocity. Conversely, a hot mainsequence star may need to spin down to reach corotation with its orbit, and transfer of angular momentum from the star to the orbit expands the orbit and reduces its angular velocity. Thus, either both the star and orbit spin up or both spin down.

To estimate the angular momentum that can be stored in a star, we consider star 1 of radius $R_{1}$ to be extended and to have a radius of gyration $k_{1} R_{1}$, and so moment of inertia $I_{\text {star }}=M_{1} k_{1}^{2} R_{1}^{2}$ and angular momentum $J_{\text {spin,1 }}$ such that

$$
\frac{J_{\text {spin }, 1}}{J_{\text {orb }}}=\frac{M_{1}\left(k_{1} R_{1}\right)^{2} M}{\left(M_{1} M_{2} a^{2}\right)} \text {. }
$$




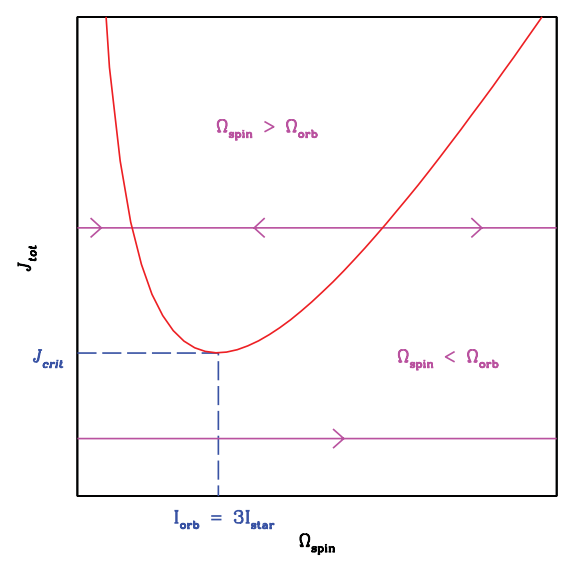

Figure 3. The total angular momentum of a binary system with angular velocity $\Omega_{\text {orb }}$ one expanded star spinning at $\Omega_{\mathrm{spin}}$. The solid curve is the tidal equilibrium. Horizontal lines are the direction of evolution when not in equilibrium. Though most detached and semi-detached binaries lie in regions where they can evolve to stable equilibrium, some contact systems and many close orbiting planetary systems are ultimately unstable.

For two $1 M_{\odot}$ main-sequence stars, with $k_{1}^{2} \approx 0.1$, and a separation of $10 R_{\odot}$

$$
\frac{J_{\text {spin }, 1}}{J_{\text {orb }}} \approx 0.1 \times\left(\frac{1}{10}\right)^{2} \times 2=2 \times 10^{-3}
$$

and this we might neglect. However, for a $5 M_{\odot}$ giant with $k_{1}^{2} \approx 0.2$ for its envelope, a core of $M_{\mathrm{c}}=0.6 M_{\odot}$, envelope radius of $R=300 R_{\odot}$, almost filling its Roche lobe when $a=500 R_{\odot}$ and a companion of $0.5 M_{\odot}$,

$$
\frac{J_{\text {spin }, 1}}{J_{\text {orb }}} \approx 0.2 \times\left(\frac{300}{800}\right)^{2} \times \frac{4.4 \times 5.5}{5.0 \times 0.5} \approx 0.27
$$

which becomes significant. Thus, the orbit of a system can be very much reduced by the time that a giant fills its Roche lobe. Indeed for large mass ratios, spinning up the star can take more angular momentum than is available in the orbit. Close binary systems with extreme mass ratios are most prone, and planets that can spin up their host star are very vulnerable. The total angular momentum of a system with one expanded star is

$$
J_{\text {tot }}=I_{\text {star }} \Omega_{\text {spin }}+\frac{M_{1} M_{2}}{M} a^{2} \Omega_{\text {orb }} .
$$

Fig. 3 shows how this varies with the angular velocity of the star's $\Omega_{\text {spin }}$. The left branch of the equilibrium, with $3 I_{\text {star }}<I_{\text {orb }}=M_{1} M_{2} a^{2} / M$, is stable but the right, with $3 I_{\text {star }}>$ $I_{\text {orb }}$, is unstable. Our typical giant spinning more slowly than its orbit lies to the left of the stable branch. As tides spin it up, it moves to the right. However, as it evolves, tidally locked $I_{\text {star }}$ increases so that $J_{\text {tot }}$ effectively falls, and it descends the stable track. Our example system (equation 4.3 ) is dangerously close to the minimum $J_{\text {crit }}$ beyond which there is no stable equilibrium. Most contact binaries eventually suffer this fate. A hot star spinning faster than its orbit typically lies between the two equilibria and tidally evolves towards the stable branch to the left. After the discovery of 51 Peg B, Rasio et al. (1996) soon pointed out that, if the planet were able to raise tides on the star sufficient to spin it up, the planet would fall into the star because $J_{\text {tot }}<J_{\text {crit }}$. The fact that it doesn't, tells us about the strength of the tides it can raise on the star. 


\section{Consequential Angular Momentum Loss}

Most often, non-conservative mass transfer is taken to mean mass and angular momentum loss associated with the process of Roche lobe overflow itself. This needn't be due to part of the stream leaving the system at the $L_{1}$ point. Indeed, it is more likely associated with the accretion process on to the companion. When accretion is via a disc there must be some consequential angular momentum loss. Consider star 2 of radius $R_{2}$, mass $M_{2}$ and spin $\Omega$, accreting from the inner edge of a Keplerian disc. Its angular momentum $J_{2}=I_{2} \Omega \approx k_{2}^{2} M_{2} R_{2}^{2} \Omega$ while material accretes at the star's break-up spin of $\Omega_{\max }=\sqrt{G M_{2} / R_{2}^{3}}$ so that $\delta J_{2}=\delta M R_{2}^{2} \Omega_{\max }$. Then, the maximum mass that can be accreted conservatively is about $\delta M_{2} \approx k_{2} M_{2}$ because stars accreting from a disc are easily spun up. However, white dwarfs in cataclysmic variables spin slowly and primary stars in Algol systems wide enough for an accretion disc spin at most at about four tenths of break-up. Tides are not sufficiently strong in the hot Algol primaries to prevent rapid spin up and so some extra angular momentum loss is necessary. Dervişoğlu, Tout \& İbanoğlu (2010) argued that this is most likely by the excitation of a magnetic dynamo generated precisely because the star has been spun up by accretion.

Once strong large scale magnetic fields have been generated, angular momentum loss by magnetic braking (Schatzman 1962) is inevitable. Suppose mass in a stellar wind is forced to effectively corotate to some Alfvén radius $R_{\mathrm{A}}$ (Mestel 1968). Then

$$
\dot{J}=\dot{M} a_{1}^{2} \Omega+K J
$$

with

$$
K=\frac{2}{3}\left(\frac{R_{\mathrm{A}}}{R}\right)^{2}\left(\frac{R}{a}\right)^{2} \frac{M}{M_{1} M_{2}} \dot{M} .
$$

Note that $R_{\mathrm{A}} / R \geqslant 1$ always, and even this minimum is important for extreme mass ratios when $R_{1}>a_{1}$.

Similarly, rapidly spinning magnetised accretors can eject the transferred mass by a propeller effect. When the magnetic field of an accreting star is so high that the magnetic energy density in the stream is similar to its kinetic energy, the transferred material is forced to flow along the field lines. This is the case in the polars, and intermediate polars, cataclysmic variables with a highly magnetic white dwarf that accretes at the magnetic poles. In the polars, the spin of the white dwarf is locked to the orbit, but in cases where the white dwarf has been spun up beyond this, perhaps by an episode of disc accretion, material can be accelerated by the magnetic field and flung out of the system. A probable example is the cataclysmic variable AE Aquarii (Eracleous \& Horne 1996) and similar effects are expected for accreting magnetic neutron stars.

\section{Common Envelope Evolution}

Perhaps the most extreme case of non-conservative evolution is that of a common envelope phase. Paczyński (1976) described how such a process is necessary to account for the very short orbital periods of cataclysmic variables and their immediate progenitors. The white dwarf must originally have been the core of a giant of some many tens to hundreds of solar radii. Thus, a substantial amount of both angular momentum and energy must have been lost from the orbit along with the giant's massive envelope. Fig. 4 illustrates the process which remains one of the most important, but least understood, in binary star evolution. The onset of common envelope evolution follows a giant filling its Roche lobe and transferring mass to a less massive companion. The giant responds to mass loss by expanding its convective envelope, while the orbit responds by shrinking. 


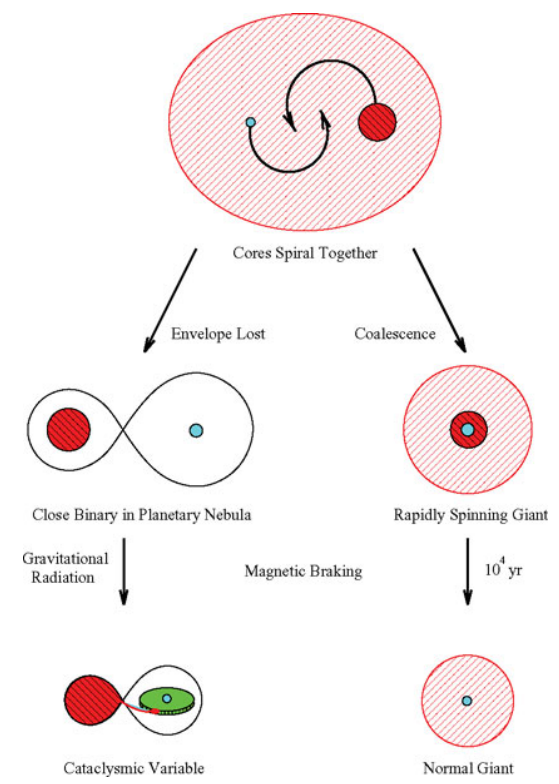

Figure 4. Common-envelope evolution. After dynamical mass transfer from a giant, a common envelope enshrouds the relatively dense companion and the core of the original giant. These two spiral together as their orbital energy is transferred to the envelope until either the entire envelope is lost or they coalesce. In the former case a close white-dwarf and main-sequence binary is left, initially as the core of a planetary nebula. Magnetic braking or gravitational radiation may shrink the orbit and create a cataclysmic variable. Coalescence results in a rapidly rotating giant which will very quickly spin down by magnetic braking.

This positive feedback means that the giant overfills its Roche lobe yet more, and the rate of mass transfer rises towards a dynamical limit. The companion certainly cannot accrete at such a rate and the material forms a common envelope.

Some wide Algol-like systems ought not to have avoided this catastrophe. Tout \& Eggleton (1988) argued that mass loss before Roche lobe overflow can be sufficiently enhanced by a companion that the mass ratio can invert well before the onset of mass transfer so that the Roche lobe grows faster than the star and mass transfer can proceed on a stable nuclear timescale. They proposed that

$$
\dot{M} \rightarrow \dot{M}\left\{1+B \min \left[\frac{R}{R_{\mathrm{L}}}, \frac{1}{2}\right]\right\}^{6}
$$

and from RS CVn systems with inverted mass ratios $B \approx 10^{4}$. Tout \& Hall (1991) went on to say that, because red giants expand on losing mass, a strong stellar wind can actually drive mass transfer. To see this, we may approximate the radius of a giant by $R_{1}=f\left(L_{1}\right) M_{1}^{-n}$, where $f\left(L_{1}\right)$ is some function of the star's luminosity $L_{1}$, which varies only as its core grows on a nuclear timescale. Typically, $n \approx 0.27$. Thus, when the timescale for mass transfer is fast compared to nuclear evolution

$$
\frac{\dot{R}_{1}}{R_{1}}=-n \frac{\dot{M}_{1}}{M_{1}}
$$

Combining this with Paczyński's formula for the Roche lobe radius,

$$
\frac{R_{\mathrm{L}}}{a}=\frac{2}{3^{4 / 3}}\left(\frac{M_{1}}{M}\right)^{\frac{1}{3}}, \quad 0<M_{1} / M_{2}<0.8,
$$


we find that when $R_{1}=R_{\mathrm{L}}$ then $\dot{R}_{1}>\dot{R}_{\mathrm{L}}$ when

$$
q=\frac{M_{1}}{M_{2}}<\frac{1+3 n}{3(1-n)},
$$

so that mass loss actually drives the giant to overflow its Roche lobe and mass transfer begins. When such mass transfer is stable, $R_{1} \approx R_{\mathrm{L}}$ and then

$$
\dot{M}_{2}=\frac{1+3 n-3(1-n) q}{(1+q)(5-3 n-6 q)} \dot{M} \text {. }
$$

If $6 q>5-3 n$, mass transfer is dynamically unstable. At lower $q, \dot{M}_{2} \approx-0.5 \dot{M}$ and about half as much mass as is lost in the wind is transferred to the companion as well.

\section{Stellar Evolution Codes}

At least two versions of the Cambridge STARS code (Eggleton 1971) can be used to evolve both components of a binary star simultaneously, taking care of mass and angular momentum transport by various physical mechanisms. These are the TWIN code (Nelson \& Eggleton 2001) and the BS code (Stancliffe \& Eldridge 2009). Versions of both are available from http://www.ast.cam.ac.uk/ stars/index.html for those who wish to experiment with them. Users are, however, warned that neither version has been written with the casual user in mind, and it would be beneficial to seek out an existing user before engaging in a project. Those listed on the web pages are often very happy to assist.

\section{Conclusions}

All binary star evolution is non-conservative to some extent. There is both direct and indirect evidence for both mass and angular momentum loss. However, there is very little agreement on the physics and its implementation. Common envelope evolution is the most extreme, but perhaps least understood, non-conservative process.

We have only picked out a few of the drivers of non-conservative evolution. There are many others. Nova eruptions on the surface of white dwarfs in cataclysmic variables occur because hydrogen ignites degenerately when a shell of $10^{-4} M_{\odot}$ or so has accumulated. The violence of the thermonuclear runaway throws off most of this accreted material, presumably along with the specific angular momentum of the white dwarf's orbit. Supernovae explosions can expel more than half the mass in a binary system, perhaps with a violent kick. This surely unbinds most systems, but the existence of X-ray binaries and binary millisecond pulsars shows that neutron stars can often be retained. Most accretion discs are associated with mass loss in jets or outflows, with typically one tenth of the accreted mass being lost. Gravitational radiation is probably the best understood mechanism for angular momentum loss, with negligible mass loss. General relativity describes precisely how fast it should operate and this is backed up by observations of many close binary systems.

We have only briefly touched on the uncertainties of stellar mass loss rates. Even for single stars it is mass loss that determines the end points of stellar evolution. But when does a star lose most of its mass? Is it a relatively gradual process that slowly increases over its evolution or is it all lost at the end in an asymptotic giant's superwind (Vassiliadis \& Wood 1993). In a binary, it matters how fast the material leaves the star because, if it is slow enough, a significant fraction of the stellar wind can be accreted by a companion as 
it orbits through the expanding gas. This is just yet another uncertainty in the evolution of binary stars still to be fully understood.

CAT is very grateful to Churchill College Cambridge for his Fellowship and to the STFC for funding his attendance at this symposium.

\section{References}

Crawford, J. A. 1955, ApJ, 121, 71

Dervişoğlu, A., Tout, C. A., \& İbanoğlu, C. 2010, MNRAS, 406, 1071

Eracleous, M. \& Horne, K. 1996, ApJ, 471, 427

Hoyle, F. 1955, Frontiers of Astronomy, (London: Heinemann)

Montez, R., Jr., De Marco, O., Kastner, J. H., \& Chu, Y.-H. 2010, ApJ, 721, 1820

Mestel, L. 1968, MNRAS, 138, 359

Morton, D. C. 1960, ApJ, 132, 146

Paczyński, B. 1976, in: P. P. Eggleton, S. Mitton S. \& J. Whelan (eds), Structure and Evolution of Close Binary Systems, Proc. IAU Symposium No. 73, (Dordrecht: Reidel), p. 75

Paczyński, B. \& Ziółkowski, J. 1967, AcA 17, 7

Rasio, F. A., Tout, C. A., Lubow, S. H., \& Livio, M. 1996, ApJ, 470, 1187

Schatzman, E. 1962, Ann. Astrophys., 25, 18

Tout, C. A. \& Eggleton, P. P. 1988, MNRAS, 231, 823

Tout, C. A. \& Hall, D. S. 1991, MNRAS, 253, 9

Vassiliadis, E. \& Wood, P. R. 1993, ApJ, 413, 641

\section{Discussion}

O. De Marco: Common envelope simulations/theory indicate that envelope mass does not accrete on to the secondary star. However, X-ray observations of post common envelope binaries (Montez et al. 2010) are consistent with a spun up secondary (which has an X-ray bright corona generated by the spin). What is your opinion about accretion on to the secondary?

C. Tout: The length of the dynamical phase is so short that there is no time to accrete. So, it would follow that there is no accretion. However, I think that if the CE interaction evolved over a longer time, there could be time for accretion. The observations you mention may argue for such a longer phase.

P. Harmanec: Is there evidence for much mass loss from Algol systems?

C. Tout: There is no quantitative measure of mass loss from Algol systems, all of which are observed now in a slow phase of mass transfer. I expect mass loss from the system at a rate similar to the mass transfer rate could easily escape unnoticed. Some evidence might be forthcoming from orbital period variations but the only star to show a measurable long term change is $\mathrm{U}$ Cep, the mass ratio of which places it so close to dynamical instability that only a very small systemic mass loss can drive substantial mass transfer and so it is hard to distinguish between nuclear evolution and wind-driven mass transfer.

G. Peters: The amount of mass lost in an Algol system is unknown but we can identify a number of sites of mass loss that include, (1) a splash region caused by the gas stream impact in direct-impact systems, (2) the commonplace mass loss near phase 0.5 and (3) bipolar flows. Furthermore, Walter van Rensbergen has shown that hot accretion spots can drive mass out of the system. Given the uncertainties in the area over which the mass loss occurs and the outflow velocities (we usually derive a projected velocity), the extent of the total mass loss remains an open question. 